\title{
Numerical simulations of beam propagation through optical turbulence for high-altitude platform crosslinks
}

\author{
Joachim Horwath $^{(1)}$, Nicolas Perlot ${ }^{(1)}$, Dirk Giggenbach ${ }^{(1)}$, \\ Ralf Jüngling ${ }^{(2)}$ \\ ${ }^{(1)}$ German Aerospace Center (DLR), Institute of Communications and Navigation, \\ Weßling, Germany, \\ ${ }^{(2)}$ Department of Computer Science and Engineering, OGI School of Science \& Engineering, \\ Oregon Health \& Science University, Portland, Oregon
}

\begin{abstract}
In this paper we introduce a simulation method for modelling clear-air atmospheric turbulence effects for long horizontal stratospheric paths. Divergence angles of several hundred microradians in combination with link distances up to $800 \mathrm{~km}$ require to adapt the appropriate resolution of the transverse optical field along the path. For this purpose, we implemented a propagation method in Cartesian coordinates. We choose two reference scenarios for high-altitude platform crosslinks and discuss the influence of simulation parameters to the derived results. Finally a method for computation of temporal IM/DD-time signals form simulated intensity matrices is presented.
\end{abstract}

Keywords: numerical simulation, index of refraction effects, High Altitude Platform, long stratospheric paths, phase screen method

\section{INTRODUCTION}

Many questions concerning the propagation of Laser light in the turbulent atmosphere cannot be answered analytically. In order to design appropriate communication systems a certain number of important parameters at the receiver, (e.g., scintillation index, intensity correlation length, angle of arrival) can be derived from computer simulation results. A simulation helps identifying the important factors of an experiment before actual measurements are made. In particular, several technical problems remain for the channel measurements of long stratospheric paths which are planned to sustain broadband optical links between future High Altitude Platform (HAP) telecommunication systems. Such HAP systems can provide broadband services to the customer by combining advantages of terrestrial and satellite systems. Optical inter-platform links will be used to build up a stratospheric back haul network independent from terrestrial ground network.

Our simulation methodology, as outlined in section 2, is based on the well known split-step approach to numerically solving the parabolic wave equation. We explain how occasional resampling of the field makes this approach feasible for long-range propagation scenarios. Even though the complex optical field at the receiver can be obtained from simulation, here we confine our evaluations to the resulting intensity matrix. Phase information is not considered because we are focusing on intensity modulated systems with direct detection at a wavelength $\lambda$ of $1064 \mathrm{~nm}$. The

Author Contact: joachim.horwath@dlr.de

Joachim Horwath, Institute of Communications and Navigation,

German Aerospace Center (DLR), Site Oberpfaffenhofen, D-82234 Wessling, Germany

Copyright 2004 Society of Photo-Optical Instrumentation Engineers.

This paper was published in Proc. of SPIE Vol. 5338B and is made available as an electronic reprint with permission of SPIE. One print or electronic copy may be made for personal use only. Systematic or multiple reproduction, distribution to multiple locations via electronic or other means, duplication of any material in this paper for a fee or for commercial purposes, or modification of the content of the paper are prohibited. 
parameters used to model stratospheric turbulence are given in section 3. Numerical considerations are discussed in section 4. The required number of random phase modulators (phase screens), by use of which turbulence effects are modelled, is part of those considerations. Due to the curvature of the earth, the height profile of optical links between two HAPs is parabolically shaped. This leads to minimal path heights in the middle of the path, when the two HAPs are at the same altitude. In order to guarantee an availability of 100 percent for the stratospheric optical crosslinks, the minimal link height of the path must be above the cloud layer. For midlatitude locations the cloud boundary is at an altitude of $13 \mathrm{~km}$ [1]. With this constraint given, an optical communication crosslink between two HAPs at $25 \mathrm{~km}$ altitude for example, can reach a distance of $784 \mathrm{~km}$.

In this paper we consider two different scenarios, each involving two HAPs (the transmitter and the receiver) positioned at an altitude of $20 \mathrm{~km}$ (Fig. 1). The first scenario, featuring a propagation distance of $300 \mathrm{~km}$, is designed to qualify for so called "weak fluctuation" conditions by using a "best case"-turbulence profile defined in section 3 . The second scenario (propagation distance $600 \mathrm{~km}$ ) is an instance of "strong fluctuation" conditions which is simulated with "worstcase" turbulence conditions. The height profile of these two scenarios could be seen in (Fig. 2). The minimal path heights are $18.2 \mathrm{~km}$ for Scenario 1, and $13 \mathrm{~km}$ for Scenario 2.

The divergence angle is one of the most important parameters in the design of optical free space communications systems. For typical transmitter aperture sizes of 10 to $20 \mathrm{~cm}$, a diffraction limited divergence angle in the range of 5 to $10 \mu \mathrm{rad}$ would result in the highest attainable antenna gain. This would impose high requirements on the pointing and tracking (PAT) system. For inter-platform links, the selection of an appropriate divergence angle depends mainly on the platform base motion disturbance and vibration spectrum, which is mostly influenced by station keeping propellers. A beam divergence of several hundred $\mu \mathrm{rad}$ is suitable for optical inter platform links [1]. Here we have chosen a collimated Gaussian beam with a full $1 / \mathrm{e}^{2}$ intensity-divergence angle of $500 \mu \mathrm{rad}$. Note that, with such a high divergence angle and such long propagation paths, the Gaussian beam wave is close to a spherical wave in terms of scintillation. Increasing its divergence leads to little difference in the scintillation.

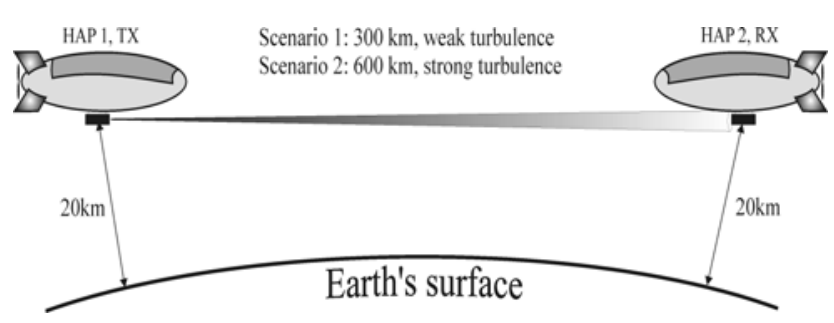

Fig. 1: Setup of scenarios used for simulation

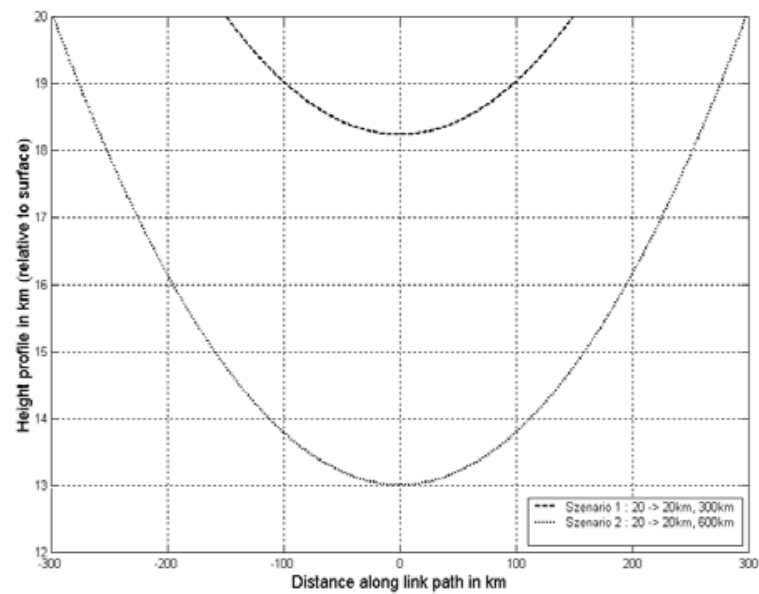

Fig. 2: Height profiles of considered scenarios

Besides the formation of intensity speckles observable in a cross-sectional plane of the beam, scintillation is also characterized by the wander of the beam due to turbulent cells large in comparison to the beam width. Owing to the low turbulence of the upper atmosphere, the latter effect is weak and therefore, the evaluation of our simulations will focus on the intensity redistribution due to smaller turbulent scales.

The simulation method enables us to observe at the receiver only a finite extent of the optical field. In particular, we wish to estimate the scintillation near the beam axis. In HAP links, the pointing and tracking system will make the beam wander around the receiver, and therefore the off-axis scintillation has to be investigated if it is significantly higher than 
the on-axis scintillation. For highly divergent beams, e.g. with a 500- $\mu$ rad full divergence angle, the difference between on- and off- axis scintillation indices $\sigma_{I}^{2}=\left\langle I^{2}\right\rangle /\langle I\rangle^{2}-1$ is negligible [25].

For these types of scenarios, a method to transfer the spatial statistics of the simulated intensity distribution at the receiver to a temporal direct-detected signal is introduced in (5).

\section{SIMULATION METHODOLOGY}

For the problem of "clear air" atmospheric optical propagation a number of simplifications can be applied to Maxwell's equations. Absence of depolarization effects allows the consideration of a scalar optical field which leads to a stochastic Helmholtz equation; small angle scattering allows for further simplification to the parabolic equation

$$
\partial_{z} u=\frac{i}{2 k}\left(\partial_{x}^{2}+\partial_{y}^{2}\right) u+i k n_{1} u
$$

The complex quantity $u(\mathbf{r})$ denotes the slowly varying envelope of the scalar electromagnetic field; $k$ is the wave number and $n_{1}(\mathbf{r})=n(\mathbf{r})-\overline{n(\mathbf{r})}$ is the local deviation of the refractive index from its ensemble average. Note, that in (1) we also neglect time dependency of the medium. Without the second term, which models stochastic phase perturbations due to the atmosphere, (1) is the parabolic wave equation, one of whose solutions are Gaussian beams [2]. In the turbulent regime, the stochastic process $n_{1}$ is governed by the well known power law $\overline{\left[n_{1}\left(\mathbf{r}_{0}\right)-n_{1}\left(\mathbf{r}_{0}+\mathbf{r}\right)\right]^{2}} \sim r^{2 / 3}$ within the so called inertial subrange $l_{0}<r<L_{0}$ [3] and is assumed to obey Gaussian statistics.

The split-step approach to numerically simulating atmospheric propagation amounts to partitioning the propagation distance into "reasonably sized" sub-distances $\Delta z_{i}=z_{i}-z_{i-1}, i=1 \ldots N, z_{0}=0$ and to alternately simulate the effects of both terms in (1) individually for each $\Delta z_{i}$. "Reasonably sized" here means that $\Delta z_{i}$ is, on the one hand, small enough so that the "split-step approximation"

$$
u\left(x, y, z_{i}\right) \approx \exp \left(\frac{\Delta z_{i}}{2} \frac{i}{2 k}\left(\partial_{x}{ }^{2}+\partial_{y}^{2}\right)\right) \exp \left(\int_{z_{i-1}}^{z_{i-1}+\Delta z_{i}} i k n_{1} d z\right) \exp \left(\frac{\Delta z_{i}}{2} \frac{i}{2 k}\left(\partial_{x}^{2}+\partial_{y}^{2}\right)\right) u\left(x, y, z_{i-1}\right)
$$

of the formal solution to (1) is justified and, on the other hand, large enough so that the realizations of the stochastic phase perturbations (which we call phase-screens and note PS henceforth) can be generated independently for each $\Delta z_{i}$. This algorithm has been thoroughly described in the literature, details can be found in, e.g., [4][5][6][7]. Here we merely have space to outline how the specific problems associated with long-range beam propagation are dealt with in our simulation ${ }^{1}$.

The complex field $u\left(x, y, z_{i}\right)$ is represented by a finite number of sample values as obtained by a regular sampling in $\mathrm{X}$ and Y-dimension, that is, by a grid of sample values $u\left(k \Delta x, l \Delta x, z_{i}\right), k=1 \ldots M, l=1 \ldots M$. For $z_{0}$ the grid simply bears the sampled transmitter beam profile; the values at $z_{i}, \mathrm{i} \geq 1$, are subsequently obtained from the values at $z_{i-1}$ as sketched above. If computational resources were unlimited, one would always choose a grid size large enough to cover the entire beam profile with a spatial sampling rate appropriate to accurately resolve all small-scale modulations of the field. In long-range beam propagation scenarios, however, this turns out to be infeasible for the beam diameter becomes very large due to diffraction. One solution which has already been proposed by Rubio et al. [8], is to sacrifice coverage of the

\footnotetext{
${ }^{1}$ Our simulation is based on PILab (short for "Propagation and Imaging Lab"), a Matlab-based programming environment developed at DLR to support simulation of optical free-space communication scenarios [9].
} 
full beam cross section in favor of maintaining a sampling interval that resolves atmospherically induced modulations of the field on all scales equal and below the receiver aperture diameter. The order of magnitude of an appropriate sampling interval must not become larger than $l_{0}$, the lower bound of the inertial subrange of turbulence. We maintained $\Delta x \leq l_{0} / 3$ in our simulations.

In order to simulate beam propagation effectively, the sampling interval gets adapted according to the diffractive beam spread. Like Rubio et al., we resample the field at those propagation distances $z_{i}$ where the next propagation step would render the sampling rate insufficient without resampling (see Fig 3). The grid size stays the same, so the extent of the field represented by the grid gets reduced in a resampling step (from some value $X_{i}^{\prime}$ to $X_{i}$, cf. Fig 1). Since we use an FFT-based propagation algorithm entailing periodic boundary conditions, we finally multiply the resampled field with a super-Gaussian damping function to avoid spurious "self-interference"2 in subsequent propagation steps. Unlike Rubio et al., who use spherical coordinates, we carry out calculations in Cartesian coordinates and employ a coordinate transformation of the paraxial diffraction formula (referred to as Talanov transform in [4] and [9]) to adapt the sampling interval.

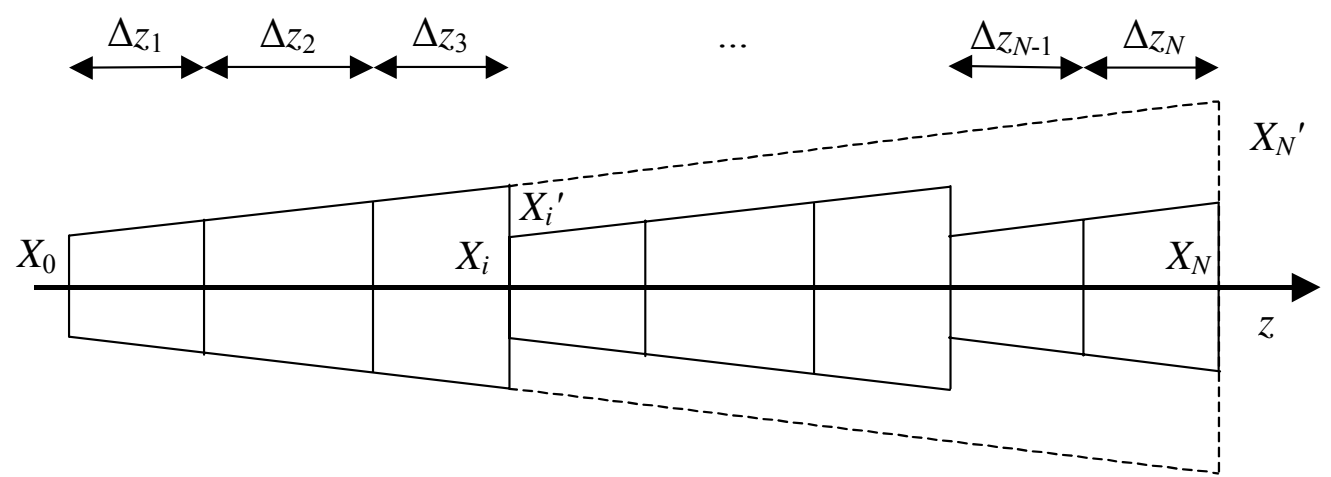

Fig. 3: Step-wise simulation with occasional resampling of the field

\section{TURBULENCE PARAMETERS IN THE STRATOSPHERE}

In order to get realistic simulation results, reliable values for the physical stratospheric conditions are required. Intensive and reliable measurements of the turbulence in the stratosphere are rare. For the index-of-refraction structure constant $\mathrm{C}_{\mathrm{n}}{ }^{2}$, we used profiles based on several reported measurements from balloon ascents and other experiments [10]-[24]. From those measurement data, one can define a best- and a worst-case $\mathrm{C}_{\mathrm{n}}{ }^{2}$ profile for stratospheric altitudes:

$$
C_{n}^{2}(h)=a \cdot 10^{-h / 8000} \quad 13 \mathrm{~km}<h<25 \mathrm{~km}
$$

$$
\begin{array}{ll}
a=1.5 \times 10^{-16} \mathrm{~m}^{-2 / 3} & \text { for the best case } \\
a=8 \times 10^{-16} \mathrm{~m}^{-2 / 3} & \text { for the worst case }
\end{array}
$$

where $h$ is the altitude in meters.

The tropopause is assumed to remain below the altitude of $13 \mathrm{~km}$. The stratospheric fall-off of these boundary profiles does not depend on the tropopause wind speed. This dependence, which is incorporated in the Huvnagel-Valley (H-V) model (see for example [25]), is actually in disagreement with some reported data [26]. Also note, that the stratification of the turbulent upper-atmosphere as well as the possible horizontal variability of $C_{n}^{2}$, are not modeled by such simple profiles. Fig. 4 shows the $C_{n}{ }^{2}$ profiles used in our simulations along with different $\mathrm{H}-\mathrm{V}$ profiles characterized by the mean tropopause wind.

\footnotetext{
${ }^{2}$ Other authors call the effect "aliasing"; it actually is the analog to aliasing in the spatial domain.
} 
The parabolic height profile (Fig. 2) leads to a maximum $C_{n}^{2}$ value at the middle of the path. Since $C_{n}^{2}(h)$ features a constant logarithmic slope, the ratio of maximum to minimum $C_{n}^{2}$ value depends only on the link distance, the height of the transceivers having a negligible influence. This $C_{n}^{2}$ ratio is shown in Fig. 5 as a function of link distance. It can be seen that for distances up to $300 \mathrm{~km}$, a constant $C_{n}^{2}$ may be assumed.

In order to render one scenario in the weak fluctuation regime and the other in the strong fluctuation regime, the $300 \mathrm{~km}$ link was studied with the best case turbulence profile ("scenario 1"), while the $600 \mathrm{~km}$ link was studied with the worst case profile ("scenario 2").

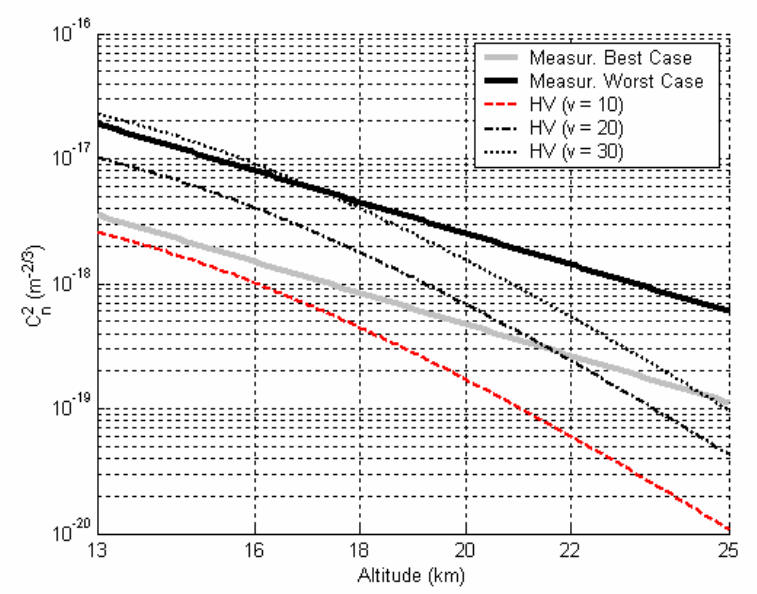

Fig. 4: Stratospheric $C_{n}^{2}$ profiles. The proposed best- and worst- case profiles are compared to the parametric Huvnagel-Valley model. The tropopause wind of the H$\mathrm{V}$ plots takes on the values $v=10,20$ and $30 \mathrm{~m} / \mathrm{s}$.

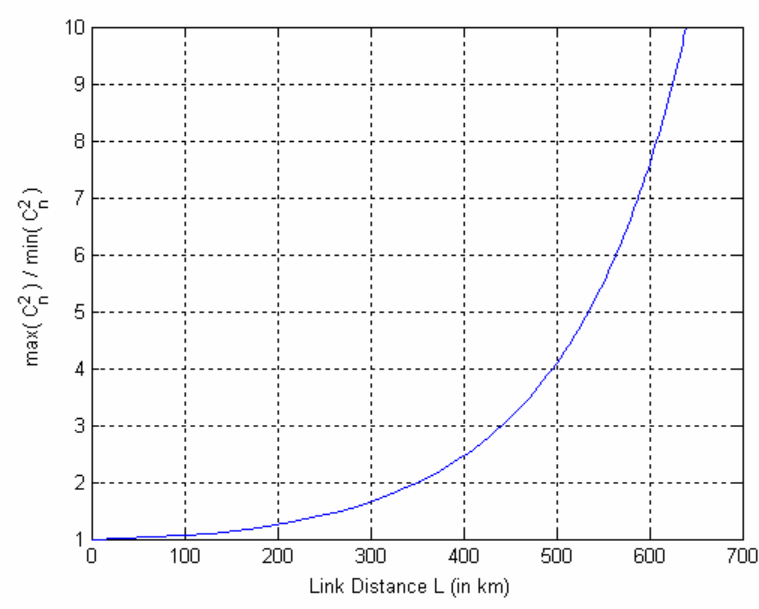

Fig. 5: Ratio of the maximum to the minimum $\mathrm{C}_{\mathrm{n}}^{2}$ as a function of link distance. The altitudes of the transmitter and receiver are $20 \mathrm{~km}$, and $\mathrm{C}_{\mathrm{n}}^{2}(\mathrm{~h})$ follows equation (3).

Besides the index-of-refraction structure constant, assumptions about the intertial subrange, that is, about its lower and upper bound $l_{0}$ and $L_{0}$, must also be made. The parameters $l_{0}$ and $L_{0}$ are also known as the inner and outer scale of turbulence. For the inner scale $l_{0}$, no general profile of the height dependency is available for the stratosphere so far. From experiments in the lower stratosphere [27], a scale size in the order of several $\mathrm{cm}$ was reported. A constant value of $5 \mathrm{~cm}$ was chosen for the inner scale $l_{0}$. Values for the outer scale $L_{0}$ in the upper atmosphere are still controversial. Some models give values of several hundred meters [28], [29]. Barat and Bertin derived $L_{0}$ values of about 10 to 100 meters from stratospheric balloon trials [30]. Bearing in mind that large outer scales have little effect on scintillation and that beam wander is not considered, $L_{0}$ has been freely set to 100 meters in our simulations.

The refractive-index power spectrum used for phase screen generation is the so-called modified spectrum defined as [25]:

$$
\Phi_{n}(k)=0.033 C_{n}^{2}\left[1+1.802\left(\kappa / \kappa_{l}\right)-0.254\left(\kappa / \kappa_{l}\right)^{7 / 6}\right] \frac{\exp \left(-\kappa^{2} / \kappa_{l}^{2}\right)}{\left(\kappa^{2}+\kappa_{0}^{2}\right)^{11 / 6}}, \quad 0 \leq \kappa<\infty
$$

where $\kappa$ is the wave number, $\kappa_{1}=3.3 / l_{0}$ and $\kappa_{0}=1 / L_{0}$.

\section{PARAMETERS OF NUMERICAL SIMULATION}

The maximum sampling interval size $\Delta x_{\max }$ was set to $1 \mathrm{~cm}$. We used a grid size M of 1024 by 1024 sample points. As it turns out, the entire turbulence spectrum cannot be modeled by a sampling grid of this size. Indeed, cell sizes on the order of $L_{0}$ are always larger than the matrix extent. It has been reported in [7] that the effect of outer scale is very small, when its value is within about an order of magnitude larger than the Fresnel length $\sqrt{L / k}, k$ being the wave number 
$2 \pi / \lambda$ and $L$ the propagation distance. For our HAP scenarios, the Fresnel length is between 20 and $40 \mathrm{~cm}$ in the NIR. Thus, the scale range covered by the grid includes all turbulence scales effectively distorting the optical wave field.

The extent of the final matrix is about 10 meters by 10 meters and the valid area (excluding the damped region) is about 8 meters by 8 meters. Although the valid area is much bigger than the typical speckle size (which is on the order of the Fresnel length or less), an accurate estimation of the intensity statistics through the assumption of ergodicity cannot be achieved from a single simulation realization. Indeed, turbulent cells of relatively large sizes create localized regions of high intensity speckles in the receiver plane. Those regions may not always be present in the final intensity matrix.

As explained in section 2, two competing factors affect the partitioning of the propagation path into sub-distances $\Delta z_{\mathrm{i}}$ : To allow for generation of stochastically independent phase screens, $\Delta z_{i}$ must be greater than the outer scale of turbulence $L_{0}$. On the other hand, to adhere to the line of argument that led to the split-step solution, $\Delta z_{\mathrm{i}}<<k l_{0}^{2}$ should hold, ensuring that phase distortions along $\Delta z_{i}$ cannot give rise to amplitude distortions within the same sub-distance. This latter requirement, however, leads to a high number of sub-distances, too high to render a computationally feasible simulation setup for long-range propagation scenarios. Therefore, in this section we investigate how far the upper bound on $\Delta z_{i}$ may be relaxed without compromising the validity of certain intensity statistics of the propagated optical field. Other authors have proposed heuristic upper bounds on sub-distances involving a kind of "local Rytov index"[26]:

$$
\sigma_{1}{ }^{2}\left(\Delta z_{\mathrm{i}}\right)=2.25 k^{7 / 6} \int_{\eta=0}^{\Delta z_{i}} C_{n}^{2}(\eta)\left(\Delta z_{i}-\eta\right)^{5 / 6} d \eta
$$

E.g., Martin \& Flatte suggested an implicitly posed upper bound by [7]

$$
\sigma_{1}^{2}(\Delta \mathrm{z})=0.1
$$

or $\sigma_{1}^{2}\left(\Delta z_{\mathrm{i}}\right) \leq 0.1 \sigma_{1}^{2}(L)$ for the case of very weak turbulence $\left(\sigma_{1}^{2}(L)<0.1\right)$. Under a constant $C_{\mathrm{n}}^{2}$, this latter condition guarantees a minimum of 4 phase screens.

The Rytov index of long stratospheric links is usually larger than 0.1. Applying the common rules for the PS placement means that a few phase screens can model stratospheric path length of several hundred kilometers. The Rytov indices of the 2 scenarios described earlier are 0.72 and 44.2 , respectively.

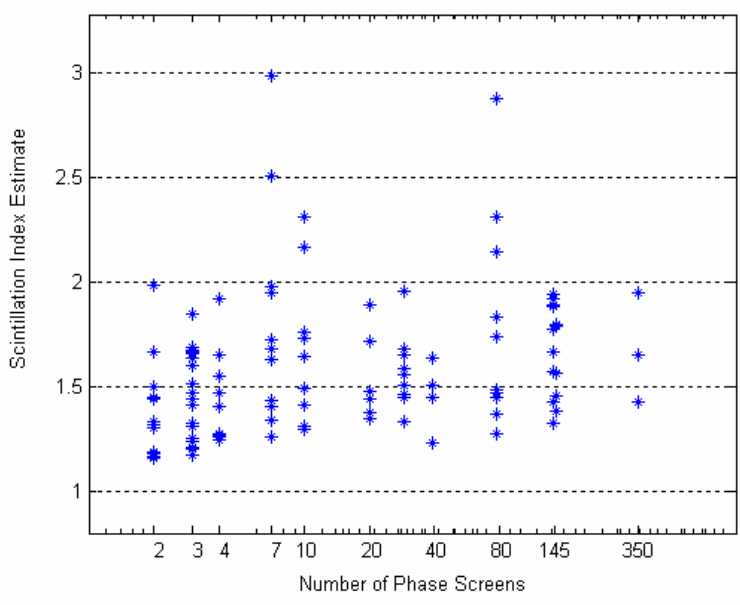

Fig. 6: SI of Scenario 2 as a function of the number of phase screens.

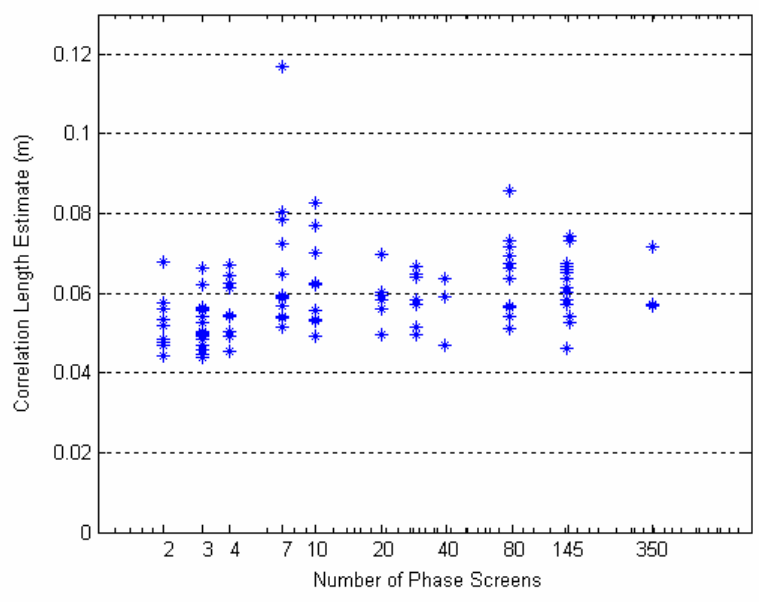

Fig. 7: Correlation length of Scenario 2 as a function of the number of phase screens.

Scenario 1 has an almost constant $\mathrm{C}_{\mathrm{n}}^{2}$ (section 3) and, according to (6), a number of 3 PS is sufficient. Following [7] again, the number of PS for Scenario 2 is approximately 30. Several propagations have been done for this scenario with different numbers of PS introduced in the optical path. The inter-PS distances $\Delta z_{i}$ were calculated so that $\sigma_{1}{ }^{2}\left(\Delta z_{i}\right)$ has the same value for all $\Delta \mathrm{z}_{\mathrm{i}}$. This leads to shorter $\Delta \mathrm{z}_{\mathrm{i}}$ near the middle of the path. For a given number of PS, several independent realizations have been done. From each resulting intensity matrix, the scintillation index (SI) and the 
correlation length as defined in section 7, have been estimated. SI estimates were computed as the mean square of the normalized intensity samples in the valid region. As for the covariance function, it was estimated from an unbiased estimate of the 2D-autocorrelation of the intensity matrix, which in turn has been averaged over the polar angle to get a one-dimensional function. Results are displayed in Fig. 6 and Fig. 7. As expected, we observe a high standard deviation of the estimates. It could have been hoped that the increase of the number of PS would reduce, to some extent, the variance of the estimates. Yet, no variation of this variance could be clearly observed as the number of PS increases. Besides, for any number of PS, no radical changes in the PDF shape could be observed, even for just a few PS. It follows that the required number of PS used to model the optical path does not need to be governed by strict rules.

\section{COMPUTATION OF TEMPORAL VECTORS FROM INTENSITY MATRICES}

In order to investigate the intensity modulated communication channel, we extract from the simulation matrices a onedimensional vector characterising the received signal of a direct-detection system. This vector can then be used to display various statistical functions or may serve as input to channel coding or interleaving simulations [31].

For the extraction of a direct-detected signal, there are two requirements. Firstly the information contained in the matrix must be representative of the scintillation statistics. As encountered in section 4, even if the correlation length is small compared to matrix size, an accurate statistical estimation cannot be guaranteed from the result of a single realization. Accuracy requires averaging over several independent realizations. Secondly, the generation of the vector relies on the "frozen turbulence" hypothesis. According to this hypothesis, turbulent motion of the medium can be neglected (in comparison with the wind-driven motion of the medium as whole) in modelling the evolution of the intensity pattern at the receiver. We further assume that the wind transverse to the direction of propagation is predominant The temporal vector is generated from the random walk of an integrating receiver aperture over the spatial intensity matrix. The sampling time is given by the spatial sampling interval multiplied by the mean transverse wind speed. It is important that the path taken by the moving aperture does not introduce additional correlation. The aperture is moved along continuous straight segments (see Fig 8). End points of the segments are generated with independently Gaussian distributed $x$ and $y$ coordinates. In this way, the directions of the path segments are uniformly distributed over $[0,2 \pi]$ and more information can be retrieved from the anisotropic intensity field. Due to circular field dampers used for our simulation approach (see section 2), the valid matrix area at the receiver has a circular finite extent. Random end points, which are generated out of the valid area by the random number generator, are set to the border. The standard deviation $\sigma$ of each coordinate of the random points should be chosen so that the points within this circular valid area have the same probability to be considered. We found that when the ratio of the matrix radius to the standard deviation equals 0.70, we obtain a quasi uniform evaluation of the grid points. Fig. 9 shows the distribution of evaluated matrix elements over the cross section of the intensity matrix which is approximately flat. A point of the matrix is evaluated, each time the point is on the path of a very long random vector. The first part of this vector is displayed in Fig 8 .

For a beam propagating $300 \mathrm{~km}$ with a divergence angle of $500 \mu \mathrm{rad}$, the ratio of the matrix extent to the beam diameter is about 0.05 in the receiver plane. We can therefore assume that the mean intensity is constant over the matrix extent of our simulations and that the matrices present only statistics of the center of the Gaussian beam. 


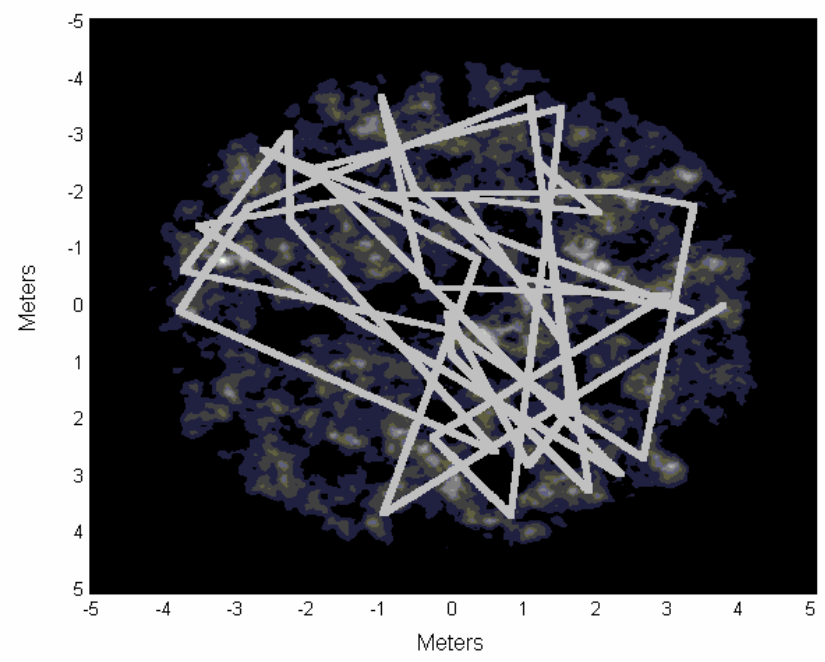

Fig. 8: Creation of a time vector. In this example, the thickness of the line corresponds to the size of the integrating aperture.

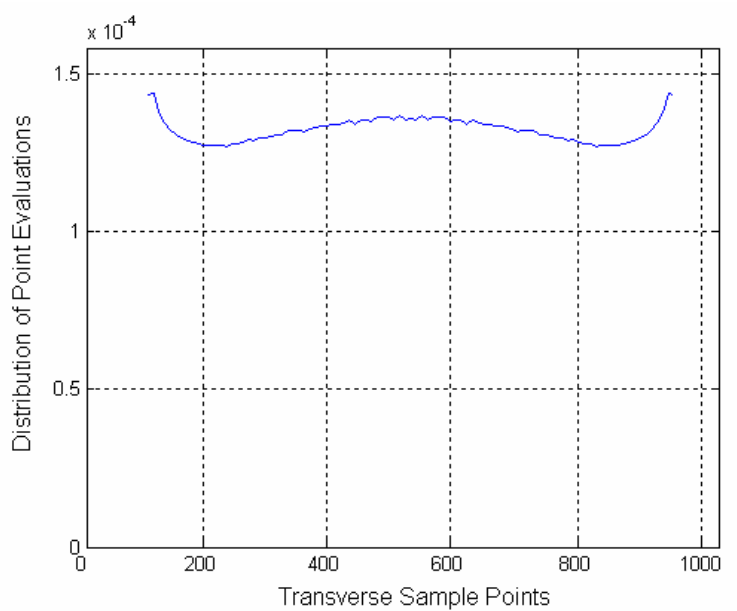

Fig. 9: Distribution of the evaluated matrix elements over the cross section of the intensity matrix.

Firstly, vectors have been extracted from the simulation results of scenario 1 and scenario 2 by using a point receiver. Each resulting temporal intensity vectors contained 500000 samples. The number of PS comprised in the optical path were 4 and 30 for the respective scenarios. The intensity PDFs as derived from the one-dimensional vectors are displayed on Fig 10. Again, spatial ergodicity has been assumed. All curves displayed, were processed by averaging of results from three independent realizations. The corresponding SI estimates are $\sigma_{\mathrm{I}}^{2}=0.420$ and ${\sigma_{I}}^{2}=1.822$ for scenarios 1 and 2 respectively. Shapes of typical intensity PDFs of the weak- and strong- fluctuation regimes can be recognized here. Their corresponding normalized intensity covariance functions are shown in Fig. 11. Their corresponding correlation lengths were evaluated at $0.237 \mathrm{~m}$ and $0.0578 \mathrm{~m}$ for scenarios 1 and 2 respectively.

Secondly a temporal power vector was derived for scenario 2 by using a $200 \mathrm{~mm}$ aperture at the receiver. This shows a major advantage of our method, -namely aperture averaging effects are taken into account. In Fig. 10 the difference between the intensity PDF and the power PDF becomes clear. The two different PDFs of scenario 2 points out, that the probability of deep fades is decreased by aperture averaging. The probability that the received signal is below a given value equals the integration of the PDF from zero to this value. The value of the variance is also reduced by aperture averaging

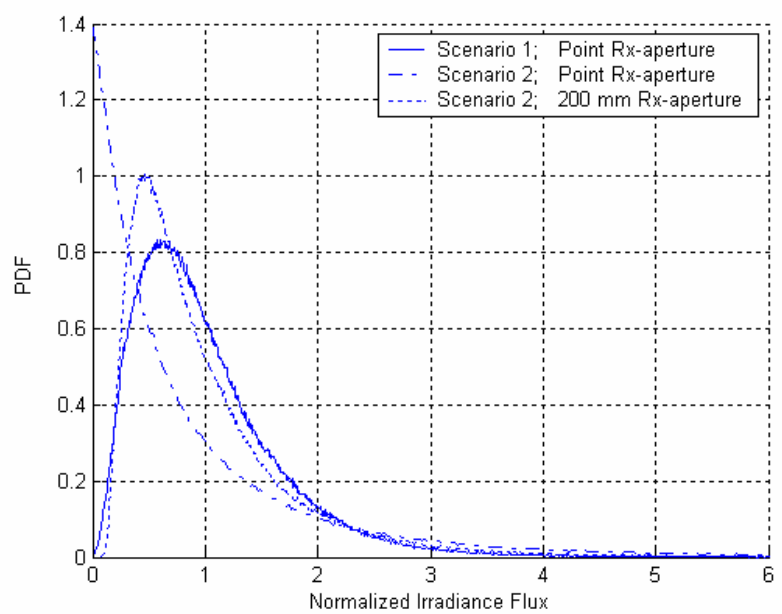

Fig. 10: PDF of a best-case $300 \mathrm{~km}$ scenario (scenario 1) with the PDF of a worst-case $600 \mathrm{~km}$ scenario (scenario 2).

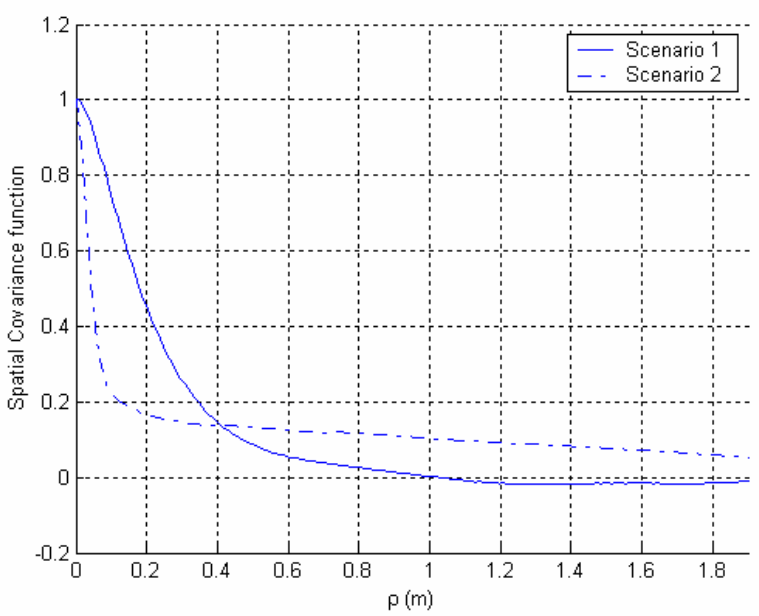

Fig. 11: Normalized intensity covariance functions for same scenario realizations as Fig. 10. 


\section{CONCLUSIONS AND OUTLOOK}

We have described a simulation method for beam propagation through an extended random media with the phase screen split-step approach. We implemented this method in Cartesian coordinates. For long horizontal links between stratospheric platforms we introduced an algorithm to keep the appropriate resolution of the transverse optical field.

Two different scenarios were analyzed. For the $300 \mathrm{~km}$ scenario the simulation results for $\mathrm{C}_{\mathrm{n}}{ }^{2}(\mathrm{~h})$ were the same as for constant $\mathrm{C}_{\mathrm{n}}{ }^{2}$. We found out that a reliable statistical evaluation of the simulated intensity distribution at the receiver necessitates to run a large number of propagation realizations. For the second scenario we tested different numbers of phase screens for our simulation method. The variance of the scintillation indices estimates derived from the different realizations was not primary influenced by the number of phase screens. The same attribute was observed for the intensity correlation length. To derive reliable values from simulations, a lot of realizations must be simulated and evaluated. The introduced method for the computation of a temporal signal vector from the simulation resuts also takes aperture averaging effects into account.

In the last section, we proposed a way to generate a direct-detected signal with a relatively arbitrary aperture. This method assumes, through the frozen turbulence hypothesis, a constant wind velocity along the propagation path. In case of turbulent layers with different velocities, a situation encountered in an optical up- or downlink with a LEO satellite, our method is not suitable to generate a temporal signal. A natural extension of our simulation methodology addressing this problem would be to simulate several propagations through a fixed set of phase screens, in succession. Prior to each subsequent simulation run, the phase screens would virtually be moved individually according to some lateral wind profile and time interval.

\section{APPENDIX}

In the following formulas we assume a continuous intensity field.

\subsection{Calculation of the scintillation index estimate from a two-dimensional intensity matrix}

Let $\rho$ a two-dimensional vector and $I(\rho)$ the intensity field. We set $I(\rho)=0$ for $\rho$ outside the valid region $V$ (see Fig. 8). The scintillation index is estimated as

$$
\hat{\sigma}_{I}^{2}=\frac{1}{V_{0}<I>^{2}} \int_{V} I^{2}(\boldsymbol{\rho}) d \boldsymbol{\rho}-1
$$

where ${ }^{\wedge}$ denotes "estimate". $\rho$ is a two-dimensional vector, $V_{0}$ is the area of the valid region $V$ and $<I>$ is the mean intensity.

\subsection{Calculation of the spatial covariance function estimate from a two-dimensional intensity matrix}

Let $W_{V}$ the window function of $V$ :

$$
W_{V}(\boldsymbol{\rho})= \begin{cases}1, & \text { if } \quad \boldsymbol{\rho} \in V \\ 0, & \text { if } \quad \boldsymbol{\rho} \notin V\end{cases}
$$

The unbiased estimate of the 2-dimensional intensity covariance function was calculated as

\footnotetext{
${ }^{3}$ When tracking a LEO satellite from earth, the relative motion between the beam and the turbulent layers is very high and dominates over wind velocities for most of the path.
} 


$$
\hat{B}_{I}\left(\boldsymbol{\rho}_{2}\right)=\frac{1}{H_{V}\left(\boldsymbol{\rho}_{2}\right)<I>^{2}} \int_{V} I\left(\boldsymbol{\rho}_{1}\right) I\left(\boldsymbol{\rho}_{1}+\boldsymbol{\rho}_{2}\right) d \boldsymbol{\rho}_{1}-1
$$

where $H_{V}\left(\boldsymbol{\rho}_{2}\right)$ represents the area of overlap of two valid regions:

$$
H_{V}\left(\boldsymbol{\rho}_{2}\right)=\int_{V} W_{V}\left(\boldsymbol{\rho}_{1}\right) W_{V}\left(\boldsymbol{\rho}_{1}+\boldsymbol{\rho}_{2}\right) d \mathbf{\rho}_{1}
$$

The 1-dimensional covariance function estimate is then given by averaging over the polar angle $\theta$ :

$$
\hat{B}_{I}(\rho)=\frac{1}{2 \pi} \int_{0}^{2 \pi} \hat{B}_{I}(\rho, \theta) d \theta
$$

and the estimate of the normalized covariance function $b_{I}(\rho)$ is

$$
\hat{b}_{I}(\rho)=\frac{\hat{B}_{I}(\rho)}{\hat{B}_{I}(0)}
$$

The correlation length is defined as the 1/e-crossing point of the normalized covariance function.

\section{REFERENCES}

1. Giggenbach, D., Purvinskis, R., Werner, M.: "Stratospheric optical inter-platform links for high altitude platforms", Proc. AIAA Conf. On Sat Communications, Montreal, May 2002

2. Kogelnik, H., Li, T.: "Laser beams and resonators". Proc. IEEE 54, p. 1312 ff, 1966

3. Clifford, S.F.: "The Classical Theory of Wave Propagation in a Turbulent Medium". In: Strohbehn, J.W. (editor): Laser Beam Propagation in the Atmosphere. Springer, 1978

4. Fleck, J. A., Morris, J. R., Feit, M. D.: "Time-Dependent Propagation of High Energy Laser Beams through the Atmosphere“. Applied Physics 10, 129-160, 1976

5. Knepp, D.L.: "Multiple phase-screen calculation of the temporal behavior of stochastic waves", Proc. IEEE 71, p. 722-737, 1983

6. Macaskill, C., Ewart, T.E.: "Computer Simulation of Two-dimensional Random Wave-Propagation", IMA J. Appl. Math. 33(1), 1-15, 1984

7. Martin, J.M., Flatté, S.M.: "Intensity images and statistics from numerical simulation of wave propagation in 3-D random media", Applied Optics 27(11), S. 2111-2126, 1988

8. Rubio, J.A., Belmonte, A., Comeron, A.: "Numerical simulation of long-path spherical wave propagation in threedimensional random media", Optical Engineering 38, 1462-1469, 1999

9. Jüngling, R.: "Simulation gerichteter Ausbreitung optischer Wellen in turbulenter Atmosphäre", diploma thesis, Münster, 2001. (in German)

10. Campbell, L., Elford W. G., J. atmos. terr. Phys. 52 313-320 (1990)

11. Ochs, G. R., Lawrence, R. S.: "Measurements of Laser Beam Spread and Curvature" ERL 106. WPL 6 (1970)

12. Ochs, G. R., Lawrence, R. S.: "Temperature and $\mathrm{C}_{\mathrm{n}}{ }^{2}$ Profiles Measured Ower Land and Ocean to $3 \mathrm{~km}$ Above the Surface“ ERL 251-WPL 22 U. S. Dept. of Commerce (1970)

13. Ochs, G. R., Wang, T., Lawrence, R. S., Clifford, S. F., Appl. Opt. 15 2504-2510 (1976)

14. Ochs, G. R., Hill, R. J., Appl. Opt. 24, 2430-2432 (1985)

15. Rocca, A., Roddier, F., Vernin, J., J. opt. Soc. Am. 64 1000-1004 (1974)

16. Roddier, F., Vernin, J., Appl. Opt. 16 2252-2256 (1977)

17. Roddier, F., Prog. in Opt. xix 281-376 (1981)

18. Roddier, F., Gilli, J. M., Vernin, J., J. Opt. 13 63-70 (1982)

19. Vernin, J., Roddier, F., J. opt. Soc. Am. 63 270-273 (1973)

20. Vernin, J., Roddier, F., C. r. Hebd. Séanc. Acad. Sci., Paris Serie B $280463-465$ (1975) 
21. Vernin, J., Barletti, R., Appl. Opt. 18 243-247 (1979)

22. Vernin, J., Azouit, M., J. Opt. 14 5-9 (1983a)

23. Vernin, J., Azouit, M., J. Opt. 14 131-142 (1983b)

24. Vernin, J., Pelon, J., Appl. Opt. 25 2874-2877 (1986)

25. Andrews, L., Phillips, R.: "Laser Beam Propagation through Random Media“, SPIE Press, Bellingham, 1998

26. Beland, R., R.: "Propagation through atmospheric optical turbulence", in IR/EO Handbook, Vol 2, SPIE Press Bellingham, Washington, pp 157-232 (1993)

27. Fairall, C. W., Markson, R., "Aircraft Measurements Of Temperature And Velocity Microturbulence In The Stably Stratified Free Troposphere", Proceedings of the $7^{\text {th }}$ Symposium on Turbulence and Diffusion, November 12-15, 1985, Boulder, CO

28. Weinstock, J., "Vertical turbulence diffusion in a stably stratified fluid“, J. Atmos. Sci. 35, 1022-1027 (1978).

29. Coulman, C. E., Vernin, J., Coqueugniot, Y.,, Caccia, J. L., "Outer scale of turbulence appropriate to modeling refractive index structure profiles", Appl. Opt. 27, 155-160 (1988).

30. Barat, J., Bertin, F., "On the Contamination of Stratospheric Turbulence Measurements by Wind Shear", J. Atmos. Sci. 41: 819-827 (1984)

31. Henninger, H., David, F., Giggenbach, D., Rapp, C., "Evaluation of FEC for the Atmospheric Optical IM/DD Channel", Proc. Of SPIE, Vol. 4975 